\title{
Evaluating the Utility of HealthMap as a Supplementary Surveillance Tool
}

\author{
Melinda C. Thomas*, Aaron Kite-Powell, David Atrubin and Janet J. Hamilton \\ Florida Department of Health, Tallahassee, FL, USA
}

\section{Objective}

To assess the outbreak detection utility of HealthMap, a publically available event-based biosurveillance system utilizing various internet-based media resources to identify outbreaks, at the state and local level. Results may help determine whether HealthMap should be monitored more closely as a supplementary surveillance tool.

\section{Introduction}

HealthMap collects and aggregates information from online sources to generate outbreak alerts based on disease and geographic location. This project will assess the timeliness and sensitivity of HealthMap based on outbreak posts from EpiCom, the Florida Department of Health's disease outbreak and health incident alert network.

\section{Methods}

This project compared EpiCom posts and HealthMap alerts in Florida for similarities in timing for outbreaks from January 1, 2009 to August 31,2013. The project assessed sensitivity and timeliness of HealthMap, whether both sources captured the outbreak, and which source's post was earlier. HealthMap alert date was compared to EpiCom's post date and the date the outbreak was reported to the county or state health department. Outbreaks of legionellosis, dengue, measles, and influenza and influenza-like illness (ILI) were assessed. Results for measles are described below. The variation of EpiCom post timeliness by county size was also investigated.

\section{Results}

During the study period, EpiCom reported on 13 confirmed measles outbreaks in Florida. Eight of these outbreaks were also found in HealthMap, giving HealthMap a sensitivity of $61.5 \%$ for measles. Two HealthMap measles posts based on non-U.S. media sources had no match to any EpiCom post. Based on post date, HealthMap was timelier than EpiCom for 2 of the outbreaks and just as timely for 3 of the outbreaks. However, based on the date the county health department was notified in the EpiCom posts, regular state and local surveillance detection practices are timelier for $100 \%$ of the outbreaks.

\section{Conclusions}

Preliminary analysis suggests that HealthMap is useful for surveillance activity but not for initial outbreak detection by the state and local health department due to its relatively low sensitivity and timeliness of detection. However, due to its worldwide focus, it may be useful in providing a better international view of disease activity, which could be especially valuable for Florida for situational awareness and surveillance activity due to the large amount of international travelers who visit. Additionally, there was evidence suggesting public health is being too conservative in its utilization of EpiCom. HealthMap can also be a means of informing the general public of health concerns in their area and assisting public health in recognizing what events are in the news.

\begin{tabular}{|c|c|}
\hline \multicolumn{2}{|c|}{ Alerting Timeliness for Measles Outbreaks } \\
\hline $\begin{array}{l}\text { Comparing EpiCom (EC) Post Date } \\
\text { to HealthMap (HM) Alert Date }\end{array}$ & Measl \\
\hline EC post first (outbreak count, percent) & \\
\hline 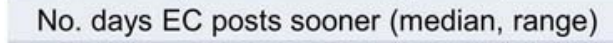 & \\
\hline Same c & $3(37.5 \%)$ \\
\hline HM alert first (outbreak count, percent) & $2(25.0 \%)$ \\
\hline No. days HM alerts sooner (median, & $2(1-3)$ \\
\hline
\end{tabular}

\section{Keywords}

Disease outbreak detection; Surveillance and alerting; Event-based biosurveillance; HealthMap

\section{Acknowledgments}

This project was supported in part by an appointment to the Applied Public Health Informatics Fellowship Program administered by CSTE and funded by a CDC Cooperative Agreement.

\section{References}

Freifeld CC, Mandl KD, Reis BY, Brownstein JS. HealthMap [Internet] 2014. Available from: http://www.healthmap.org.

*Melinda C. Thomas
E-mail: melinda.thomas2@flhealth.gov 